$4-1-2008$

\title{
Two Classes of Time-Inhomogeneous Markov Chains: Analysis of The Periodic Case
}

\author{
Attahiru Sule Alfa \\ University of Manitoba \\ Barbara Haas Margolius \\ Cleveland State University, b.margolius@csuohio.edu
}

Follow this and additional works at: https://engagedscholarship.csuohio.edu/scimath_facpub

Part of the Mathematics Commons

How does access to this work benefit you? Let us know!

Publisher's Statement

The final publication is available at Springer via http://dx.doi.org/10.1007/s10479-007-0300-3

\section{Repository Citation}

Alfa, Attahiru Sule and Margolius, Barbara Haas, "Two Classes of Time-Inhomogeneous Markov Chains: Analysis of The Periodic Case" (2008). Mathematics Faculty Publications. 245.

https://engagedscholarship.csuohio.edu/scimath_facpub/245

This Article is brought to you for free and open access by the Mathematics and Statistics Department at EngagedScholarship@CSU. It has been accepted for inclusion in Mathematics Faculty Publications by an authorized administrator of EngagedScholarship@CSU. For more information, please contact library.es@csuohio.edu. 


\title{
Transient and periodic solution to the time-inhomogeneous quasi-birth death process
}

\author{
B.H. Margolius
}

Abstract We derive the transient distribution and periodic family of asymptotic distributions and the transient and periodic moments for the quasi-birth-and-death processes with time-varying periodic rates. The distributions and moments are given in terms of integral equations involving the related random-walk process. The method is a straight-forward application of generating functions.

Keywords Quasi-birth-and-death process · Time-inhomogeneous Markov chain · Queueing model

\section{Mathematics Subject Classification (2000) $60 \mathrm{~J} 10$.} $60 \mathrm{~J} 80 \cdot 60 \mathrm{~K} 25$

\section{Introduction}

Quasi-birth-and-death processes are used to model a wide variety of applications. Among those covered in the literature are assemble to order systems [9], production lines [2], wireless communications [10] and a variety of queueing systems [14, 19]. Latouche and Ramaswami have shown that $M / G / 1$ and $G I / M / 1$ queues can be encompassed within the class of QBDs with infinite blocks [14, Chap. 13], thus vastly extending the class of problems that can be modelled with QBDs.

In many quasi-birth-and-death processes, transition rates vary periodically. For quasi-birth-and-death processes with time-varying periodic rates which meet certain stability conditions, periodic asymptotic probabilities $p_{i j}(t)$ exist. That is, the distribution $\lim _{n \rightarrow \infty} P_{l, k ; i, j}(s, n T+t)=p_{i j}(t)$ exists for $t \in[0, T)$ where $P_{l, k ; i, j}(s, n T+t)$ is the probability of the system being in level $i$, phase $j$ at time $n T+t$ given that it was in level $l$ and phase $k$ at time $s$, where $T$ is the length of the period. We present a method for computing these probabilities for level independent QBDs that requires working with integral equations over only one time period. We also derive formulae for the moments of the process as a function of time within the period. This is an extension and generalization of results obtained for the transient solution for the time-dependent Poisson queue with exponential service given in [17] and the asymptotic periodic number in an $M_{t} / M_{t} / c_{t}$ queue in [18].

As noted in [22] and references cited there, computational methods and approximation techniques involved in time-inhomogeneous queueing problems have long been regarded as challenging. In their recent paper on estimating the convergence rate of queues with nonstationary arrival and service rates, Zeifman, et al., note that "it is extremely difficult to obtain general results for arbitrary forms of the birth and death rates and therefore we must content ourselves in obtaining various types of approximations" [24]. Because of these difficulties work on queues with time-varying parameters often involves estimation schemes. See references cited in $[8,22,24]$, and [23] for a sampling of these approximation strategies.

In this paper, we present a relatively simple method for finding expressions for the transient distribution and its moments for quasi-birth-and-death (QBD) processes with timevarying rates. For those processes with periodically varying rates, we also obtain formulae for the asymptotic periodic distribution and its moments. These quantities may be computed via the numerical solution of integral equations covering only one time period. The integral equations are in terms of the quasi-birth-and-death process's related random walk 
process. The integral equations are exact, but obtaining performance measures from the formulae requires numerical solution of the integral equations and approximating or finding explicit formulae for the transition probabilities for the quasi-birth-and-death process analog of the random walk. It is hoped that these formulae will prove useful computationally in some instances, and in other cases will serve as a means for generating new approximation schemes, for confirming existing approaches and for gaining insight into the time-varying quasi-birth-and-death processes being studied.

In Sect. 2 we define a family of generating functions and state and prove our theorems for the transient case and in Sect. 2.1 for the asymptotic periodic case. In Sect. 3 we provide three examples. In Sect. 4, we describe how to numerically solve a quasi-birth-and-death process that does not have a special structure, and finally Sect. 5 is a brief conclusion.

\section{The family of generating functions}

Consider the continuous time Markov chain $\{X(t), J(t)\}$ with state space $\{\{0,1,2, \ldots\} \times\{1,2, \ldots, K\}\}$ where $K$ is a finite positive integer. $X(t)$ is referred to as the level and $J(t)$ is the phase. $K$ represents the number of phases within each level. Let us define $P_{l, k ; i, j}(s, t)=P\{X(t)=i, J(t)=$ $j \mid X(s)=l, J(s)=k\}$. The infinitesimal generator for this Markov chain may be written as

$\mathbf{Q}(t)=\left[\begin{array}{ccccc}\mathbf{B}(t) & \mathbf{A}_{1}(t) & & & \\ \mathbf{A}_{-1}(t) & \mathbf{A}_{0}(t) & \mathbf{A}_{1}(t) & & \\ & \mathbf{A}_{-1}(t) & \mathbf{A}_{0}(t) & \mathbf{A}_{1}(t) & \\ \vdots & \vdots & \ddots & \ddots & \ddots\end{array}\right]$,

where each block represents transition within levels. For example, if we define level $L_{i}=\{X(t)=i, J(t)=1,2$, $\ldots, K\}$ then $\mathbf{A}_{-1}(t)$ is a matrix that represents transitions from level $L_{i}$ to level $L_{i-1}$. This Markov chain has skip-free properties in that a transition from level $L_{i}$ to level $L_{i+k}, k>1$, will have to go through levels $L_{i+1}, L_{i+2}, \ldots, L_{i+k-1}$ before reaching level $L_{i+k}$. The off-diagonal elements of $\mathbf{Q}(t)$ are nonnegative. Its diagonal elements are all strictly negative and $\mathbf{B}(t) \mathbf{1}+\mathbf{A}_{1}(t) \mathbf{1}=\mathbf{0}$ and $\mathbf{A}_{-1}(t) \mathbf{1}+\mathbf{A}_{0}(t) \mathbf{1}+\mathbf{A}_{1}(t) \mathbf{1}=\mathbf{0}$ where $\mathbf{1}$ is a column vector of appropriate dimension whose components are all ones, and $\mathbf{0}$ is the zero vector of the same dimension. For ease of exposition, we consider the case where the period, $T$, is equal to one.

We are interested in the quantities

$p_{i, j}(t)=\lim _{n \rightarrow \infty} P_{l, k ; i, j}(s, t+n), \quad 0 \leq t<1, n$ integer.
For each $t$ in $[0,1), p_{i, j}(t), i=0,1,2, \ldots, j=1,2, \ldots, K$ is the periodic asymptotic distribution for that time within the period. In what follows, we will associate with each QBD a generalized random walk process. The periodic asymptotic distribution will exist when this associated random walk process has a negative drift.

Let $\mathbf{p}_{i}(t)=\left[p_{i, 1}(t), p_{i, 2}(t), \ldots, p_{i, K}(t)\right]$. Following Kolmogorov's approach to queues with time-varying rates, we begin with the Chapman-Kolmogorov equations [12, 13]. We can write the Chapman-Kolmogorov forward differential equations as

$\dot{\mathbf{p}}_{0}(t)=\mathbf{p}_{0}(t) \mathbf{B}(t)+\mathbf{p}_{1}(t) \mathbf{A}_{-1}(t)$,

$\dot{\mathbf{p}}_{i}(t)=\mathbf{p}_{i-1}(t) \mathbf{A}_{1}(t)+\mathbf{p}_{i}(t) \mathbf{A}_{0}(t)+\mathbf{p}_{i+1}(t) \mathbf{A}_{-1}(t)$.

We use the same system of differential equations to solve the transient problem. In this case $\mathbf{p}_{j}(t)$ represents the solution to the transient problem, but we suppress the dependence on the initial condition in the notation.

Periodic solutions to the Chapman-Kolmogorov equations will satisfy the integral equations:

$$
\begin{aligned}
\mathbf{0}= & \int_{t-1}^{t}\left(\mathbf{p}_{0}(v) \mathbf{B}(v)+\mathbf{p}_{1}(v) \mathbf{A}_{-1}(v)\right) d v, \\
\mathbf{0}= & \int_{t-1}^{t}\left(\mathbf{p}_{i-1}(v) \mathbf{A}_{1}(v)\right. \\
& \left.+\mathbf{p}_{i}(v) \mathbf{A}_{0}(v)+\mathbf{p}_{i+1}(v) \mathbf{A}_{-1}(v)\right) d v
\end{aligned}
$$

for all $i \geq 1$ and all $t$. To prove this result, simply integrate (2) and use periodicity. This result is a generalization of Theorem 1 in [17] and (1.7.3) in [19].

Recall that for a non-negative random variable, $X$ with probability distribution $p_{j}=P\{X=j\}, j=0,1, \ldots$,

$$
\begin{aligned}
\sum_{k=0}^{\infty} P\{X>k\} & =\sum_{k=1}^{\infty} \sum_{j=k}^{\infty} p_{j} \\
& =\sum_{j=1}^{\infty} p_{j} \sum_{k=1}^{j} 1=\sum_{j=1}^{\infty} j p_{j}=E[X]
\end{aligned}
$$

This result holds more generally. Define $\mathbf{q}_{i}^{(m)}(t)=$ $\sum_{j=i}^{\infty} \mathbf{q}_{j}^{(m-1)}(t)$ for $m=0,1,2, \ldots$ and $i=0,1,2, \ldots$ with $\mathbf{q}_{i}^{(0)}(t)=\mathbf{p}_{i}(t)$, then it turns out that $\mathbf{q}_{0}^{(1)}(t)=$ $\left[E\left[\mathbf{1}_{J(t)=1}\right], \ldots, E\left[\mathbf{1}_{J(t)=K}\right]\right], \mathbf{q}_{0}^{(2)}(t)=E\left[X(t) \mathbf{1}_{J(t)=1}, \ldots\right.$, $\left.X(t) \mathbf{1}_{J(t)=K}\right]+\left[E\left[\mathbf{1}_{J(t)=1}\right], \ldots, E\left[\mathbf{1}_{J(t)=K}\right]\right]$ and more generally, $(m-1) !\left[\mathbf{q}_{0}^{(m)}(t)\right]_{j}=E[(X(t)+1)(X(t)+2) \cdots$ $\left.(X(t)+m-1) \mathbf{1}_{J(t)=j}\right]$. To prove this, we require some definitions: the rising factorial [11] is given by the formula

$x^{\bar{m}}=x(x+1) \cdots(x+m-1)$,

and the (signed) Stirling numbers of the first kind [20] are given by: 
$s(k, j)=s(k-1, j-1)-(k-1) s(k-1, j), \quad k, j \geq 1$

$s(k, 0)=s(0, j)=0$;

$s(0,0)=1$.

Note that this implies $s(k, k)=1$ for $k=1,2, \ldots$ as well.

The function $m ! \mathbf{q}_{0}^{(m+1)}(t) \mathbf{1}=E\left[(X(t)+1)^{\bar{m}}\right]$, gives the expectation of the rising factorial of $X(t)+1$, which we shall refer to as the $m t h$ rising factorial moment. For $m \geq 1$, we have the following theorem:

Theorem 1 (Rising factorial moments) The rising factorial moments are given by

$$
\begin{aligned}
m ! \mathbf{q}_{0}^{(m+1)}(t) \mathbf{1} & =E[(X(t)+1)(X(t)+2) \cdots(X(t)+m)] \\
& =\sum_{k=0}^{m}(-1)^{m-k} s(m+1, k+1) E\left[X^{k}(t)\right]
\end{aligned}
$$

for $m=1,2, \ldots$

Proof The proof is by induction. From the definition for $\mathbf{q}_{0}^{(m)}$ we have

$\mathbf{q}_{0}^{(m)}(t)=\sum_{k_{m}=0}^{\infty} \sum_{k_{m}-1=k_{m}}^{\infty} \cdots \sum_{k_{2}=k_{3}}^{\infty} \sum_{k_{1}=k_{2}}^{\infty} \mathbf{p}_{k_{1}}(t)$.

Assume that

$$
\begin{aligned}
& \sum_{k_{m}=k_{m}}^{\infty} \ldots \sum_{k_{2}=k_{3}}^{\infty} \sum_{k_{1}=k_{2}}^{\infty} \mathbf{p}_{k_{1}}(t) \\
& =\sum_{k_{1}=k_{m}}^{\infty} \mathbf{p}_{k_{1}}(t)\left(\begin{array}{c}
k_{1}-k_{m}+m-2 \\
m-2
\end{array}\right) .
\end{aligned}
$$

Clearly this result holds for $m=2$. Now we show that if the result is valid for some $m$, it must hold for $m+1$. To prove this result, we change the order of summation, re-index, and use Pascal's second identity [7]:

$$
\begin{aligned}
& \sum_{k_{m}=k_{m+1}}^{\infty} \sum_{k_{1}=k_{m}}^{\infty} \mathbf{p}_{k_{1}}(t)\left(\begin{array}{c}
k_{1}-k_{m}+m-2 \\
m-2
\end{array}\right) \\
& =\sum_{k_{1}=k_{m+1}}^{\infty} \mathbf{p}_{k_{1}}(t) \sum_{k_{m}=k_{m+1}}^{k_{1}}\left(\begin{array}{c}
k_{1}-k_{m}+m-2 \\
m-2
\end{array}\right) \\
& =\sum_{k_{1}=k_{m+1}}^{\infty} \mathbf{p}_{k_{1}}(t) \sum_{k_{m}=0}^{k_{1}-k_{m+1}}\left(\begin{array}{c}
m-2+k_{m} \\
m-2
\end{array}\right) \\
& =\sum_{k_{1}=k_{m+1}}^{\infty} \mathbf{p}_{k_{1}}(t)\left(\begin{array}{c}
k_{1}-k_{m+1}+m-1 \\
m-1
\end{array}\right) .
\end{aligned}
$$

It follows that $\mathbf{q}_{0}^{(m+1)}(t)=\sum_{k=0}^{\infty} \mathbf{p}_{k}(t)\left(\begin{array}{c}k+m \\ m\end{array}\right)$ or

$m ! \mathbf{q}_{0}^{(m+1)}(t)=\sum_{k=0}^{\infty} \mathbf{p}_{k}(t)(k+1)(k+2) \cdots(k+m)$

and the result follows from the definition of the Stirling number of the first kind.

The functions $\mathbf{q}_{i}^{(m)}(t)$ satisfy systems of differential equations similar to those for $\mathbf{q}_{i}^{(0)}(t)=\mathbf{p}_{i}(t)$ given in (2). For $m \geq 0$ we have:

$$
\begin{aligned}
\dot{\mathbf{q}}_{0}^{(m)}(t)= & \mathbf{p}_{0}(t) \mathbf{B}(t)+\sum_{r=1}^{m} \mathbf{q}_{0}^{(r)}(t) \mathbf{A}_{1}(t) \\
& +\sum_{r=1}^{m} \mathbf{q}_{1}^{(r)}(t) \mathbf{A}_{0}(t)+\mathbf{q}_{1}^{(m)}(t) \mathbf{A}_{-1}(t), \\
\dot{\mathbf{q}}_{i}^{(m)}(t)= & \mathbf{q}_{i-1}^{(m)}(t) \mathbf{A}_{1}(t)+\mathbf{q}_{i}^{(m)}(t) \mathbf{A}_{0}(t) \\
& +\mathbf{q}_{i+1}^{(m)}(t) \mathbf{A}_{-1}(t),
\end{aligned}
$$

where we interpret the sum $\sum_{r=1}^{0}$ to equal zero. Since $\mathbf{q}_{0}^{(r)}(t)=\sum_{j=0}^{\infty} \mathbf{q}_{j}^{(r-1)}(t)=\mathbf{q}_{0}^{(r-1)}(t)+\mathbf{q}_{1}^{(r)}(t)$ equivalently, we may write:

$$
\begin{aligned}
\dot{\mathbf{q}}_{0}^{(m)}(t)= & \mathbf{p}_{0}(t)\left(\mathbf{B}(t)-\mathbf{A}_{0}(t)\right) \\
& +\sum_{r=1}^{m} \mathbf{q}_{0}^{(r)}(t) \mathbf{A}_{1}(t)+\mathbf{q}_{0}^{(m)}(t) \mathbf{A}_{0}(t) \\
& +\mathbf{q}_{1}^{(m)}(t) \mathbf{A}_{-1}(t) .
\end{aligned}
$$

We define a family of generating functions $P_{z}^{(m)}(t)=$ $\sum_{i=0}^{\infty} \mathbf{q}_{i}^{(m)}(t) z^{i}$. Using (3) and (4) we obtain the following differential equation for $P_{z}^{(m)}(t)$ :

$$
\begin{aligned}
\dot{P}_{z}^{(m)}(t)= & P_{z}^{(m)}(t)\left(z \mathbf{A}_{1}(t)+\mathbf{A}_{0}(t)+z^{-1} \mathbf{A}_{-1}(t)\right) \\
& +\mathbf{p}_{0}(t)\left(\mathbf{B}(t)-\mathbf{A}_{0}(t)\right)-z^{-1} \mathbf{q}_{0}^{(m)}(t) \mathbf{A}_{-1}(t) \\
& +\sum_{r=1}^{m} \mathbf{q}_{0}^{(r)}(t) \mathbf{A}_{1}(t)
\end{aligned}
$$

This differential equation has the solution,

$$
\begin{aligned}
P_{z}^{(m)}(t)= & \int_{s}^{t}\left[\mathbf{p}_{0}(v)\left(\mathbf{B}(v)-\mathbf{A}_{0}(v)\right)-z^{-1} \mathbf{q}_{0}^{(m)}(v) \mathbf{A}_{-1}(v)\right. \\
& \left.+\sum_{r=1}^{m} \mathbf{q}_{0}^{(r)}(v) \mathbf{A}_{1}(v)\right] \Phi_{z}(v, t) d v \\
& +P_{z}^{(m)}(s) \Phi_{z}(s, t)
\end{aligned}
$$

where $\Phi_{z}(s, t)$ is an evolution operator such that $\Phi_{z}(t, t)=$ I and $\frac{\partial}{\partial t} \Phi_{z}(s, t)=\Phi_{z}(s, t)\left(z \mathbf{A}_{1}(t)+\mathbf{A}_{0}(t)+z^{-1} \mathbf{A}_{-1}(t)\right)$. 
The Péano series representation [5] for $\Phi_{z}(s, t)$ is

$\Phi_{z}(s, t)=\sum_{k=0}^{\infty} \Phi_{z}^{(k)}(s, t)$,

where $\Phi_{z}^{(0)}(s, t)=\mathbf{I}$ and

$$
\begin{aligned}
& \Phi_{z}^{(k)}(s, t) \\
& =\underbrace{\int_{s}^{t} \int_{s}^{u_{k}} \cdots \int_{s}^{u_{2}}}_{k \text { integrals }}\left(z \mathbf{A}_{1}\left(u_{1}\right)+\mathbf{A}_{0}\left(u_{1}\right)+z^{-1} \mathbf{A}_{-1}\left(u_{1}\right)\right) \\
& \\
& \quad \cdots\left(z \mathbf{A}_{1}\left(u_{k}\right)+\mathbf{A}_{0}\left(u_{k}\right)+z^{-1} \mathbf{A}_{-1}\left(u_{k}\right)\right) d u_{1} \cdots d u_{k}
\end{aligned}
$$

for $k \geq 1$, (cf. Breuer, [1] p. 70 or Gikhman and Skorokhod, [3], pp. 317-319).

Let $\phi_{k}(s, t)$ be the coefficient on $z^{k}$, such that

$$
\Phi_{z}(s, t)=\sum_{k=-\infty}^{\infty} \phi_{k}(s, t) z^{k}
$$

A general formula for the $\phi_{k}(s, t)$ is

$$
\begin{aligned}
\phi_{k}(s, t)= & \sum_{j=1}^{\infty} \sum \underbrace{\int_{s}^{t} \int_{s}^{u_{j-1}} \cdots \int_{s}^{u_{2}}}_{j \text { integrals }}\left(\mathbf{A}_{i_{1}}\left(u_{1}\right) \mathbf{A}_{i_{2}}\left(u_{2}\right)\right. \\
& \left.\cdots \mathbf{A}_{i_{j}}\left(u_{j}\right)\right) d u_{1} \cdots d u_{j}
\end{aligned}
$$

where the inner sum is taken over all $j$-tuples such that $\sum_{m=1}^{j} i_{m}=k$. This follows from the fact that the $\mathbf{A}_{i}(t)$ are coefficients on $z^{i}$ in (7) above. Note that $\phi_{k}(s, t)$ is defined for all integers $k$.

The $\phi_{k}(s, t)$ have a probabilistic interpretation. $\phi_{k}(s, t)$ is a $K \times K$ matrix. Recall that $K$ is the number of phases in each level. The row $i$, column $j$ component of $\phi_{k}(s, t)$ is the probability of travelling $k$ levels to the right and from phase $i$ to phase $j$ during the time interval from time $s$ to time $t$ for a quasi-birth-and-death process with no boundary conditions. This is a generalized time-dependent random walk in which location is defined both in terms of the level and the phase. Think of a tiled stairway which extends both up and down without end. Each step has a row of $K$ uniquely colored tiles. The walker wanders aimlessly along the staircase. When the walker takes a step at time $t$, he either stays on the same step, moves up one step or moves down one step. At the same time, he can move to any of the $K$ tiles on each of the three steps, for a total of $3 K-1$ possible transitions. His movements along the staircase are governed by the rate matrices $\mathbf{A}_{-1}(t), \mathbf{A}_{0}(t)$, and $\mathbf{A}_{1}(t)$. Suppose that at time $t$ he is on a tile of color $j$ and that the $j$ th row of the $\mathbf{A}_{-1}(t)$ matrix is given by $\left[\alpha_{j, 1}^{(-1)}(t), \ldots, \alpha_{j, K}^{(-1)}(t)\right]$; the $j$ th row of $\mathbf{A}_{1}(t)$ is $\left[\alpha_{j, 1}^{(1)}(t), \ldots, \alpha_{j, K}^{(1)}(t)\right]$; and the $j$ th row of $\mathbf{A}_{0}(t)$ is $\left[\alpha_{j, 1}^{(0)}(t), \ldots, \alpha_{j, K}^{(0)}(t)\right]$ where $\alpha_{j, j}^{(0)}(t)=$ $-\sum_{i=1}^{K}\left(\alpha_{j, i}^{(1)}(t)+\alpha_{j, i}^{(-1)}(t)\right)-\sum_{i \neq j} \alpha_{j, i}^{(0)}(t)$. Given that he takes a step at time $t$, the probability that it is a step up is $-\sum_{i=1}^{K} \alpha_{j, i}^{(1)}(t) / \alpha_{j, j}^{(0)}(t)$. The probability that he goes down is $-\sum_{i=1}^{K} \alpha_{j, i}^{(-1)}(t) / \alpha_{j, j}^{(0)}(t)$. Otherwise he remains on the same step. We may compute the probability that a transition is made to a tile of particular color on any of the three steps in a similar fashion.

In most cases explicit formulae are not available for the $\phi_{k}(s, t)$. For the $M_{t} / M_{t} / 1$ queue, with arrival rate $\lambda(t)$ and service rate $\mu(t)$, the $\phi_{k}(s, t)$ are scalar functions and are given by

$$
\begin{aligned}
\phi_{k}(s, t)= & \sum_{j=0}^{\infty} \frac{\left(\int_{s}^{t} \mu(u) d u\right)^{j}\left(\int_{s}^{t} \lambda(u) d u\right)^{j+k}}{(j+k) ! j !} \\
& \times e^{-\int_{s}^{t} \lambda(u) d u-\int_{s}^{t} \mu(u) d u}
\end{aligned}
$$

for $k \geq 0$ and

$$
\begin{aligned}
\phi_{k}(s, t)= & \sum_{j=0}^{\infty} \frac{\left(\int_{s}^{t} \mu(u) d u\right)^{j-k}\left(\int_{s}^{t} \lambda(u) d u\right)^{j}}{(j-k) ! j !} \\
& \times e^{-\int_{s}^{t} \lambda(u) d u-\int_{s}^{t} \mu(u) d u}
\end{aligned}
$$

for $k<0$ and the random walk generating function is

$$
\Phi_{z}(s, t)=e^{\left.(z-1) \int_{s}^{t} \lambda(u) d u+\left(z^{-1}-1\right)\right) \int_{s}^{t} \mu(u) d u} .
$$

Figure 1 shows the relationship between the normal density with mean $\int_{s}^{t}(\lambda(u)-\mu(u)) d u$ and variance $\int_{s}^{t}(\lambda(u)+$ $\mu(u)) d u$ and the $\phi_{k}(s, t)$ for $\int_{s}^{t} \lambda(u) d u=2$ and $\int_{s}^{t} \mu(u) d u$ $=8$, perhaps providing some insight as to why the $M_{t} / M_{t} / 1$ queue behaves like a reflected Brownian motion with that mean and variance [16].

We explore the $M_{t} / M_{t} / 1$ example in more detail in Sect. 3 and provide additional examples for which explicit formulae exist. In Sect. 4 we illustrate the solution of a general QBD for which explicit formulae for the $\phi_{k}(s, t)$ are $n o t$ available. Part of the difficulty in finding explicit formulae comes from the fact that matrix multiplication is not commutative except for matrices with special structure.

Given $\phi_{k}(s, t)$, and the distribution $\mathbf{p}_{k}(s)$ for some initial time $s$, we may solve for $\mathbf{p}_{0}(t)$ numerically by solving the following Volterra equation of the second kind:

$$
\begin{aligned}
\mathbf{p}_{0}(t)= & \int_{s}^{t}\left[\mathbf { p } _ { 0 } ( v ) \left(\left(\mathbf{B}(v)-\mathbf{A}_{0}(v)\right) \phi_{0}(v, t)\right.\right. \\
& \left.\left.-\mathbf{A}_{-1}(v) \phi_{1}(v, t)\right)\right] d v \\
& +\sum_{k=0}^{\infty} \mathbf{p}_{k}(s) \phi_{-k}(s, t)
\end{aligned}
$$


Fig. 1 Graph of $\phi_{k}(s, t)$ for an $M_{t} / M_{t} / 1$ queue with $\int_{s}^{t} \lambda(u) d u=2$ and $\int_{s}^{t} \mu(u) d u=8$ shown with a normal density function with mean equal to

$\int_{s}^{t}(\lambda(u)-\mu(u)) d u=-6$ and variance equal to $\int_{s}^{t}(\lambda(u)+\mu(u)) d u=10$

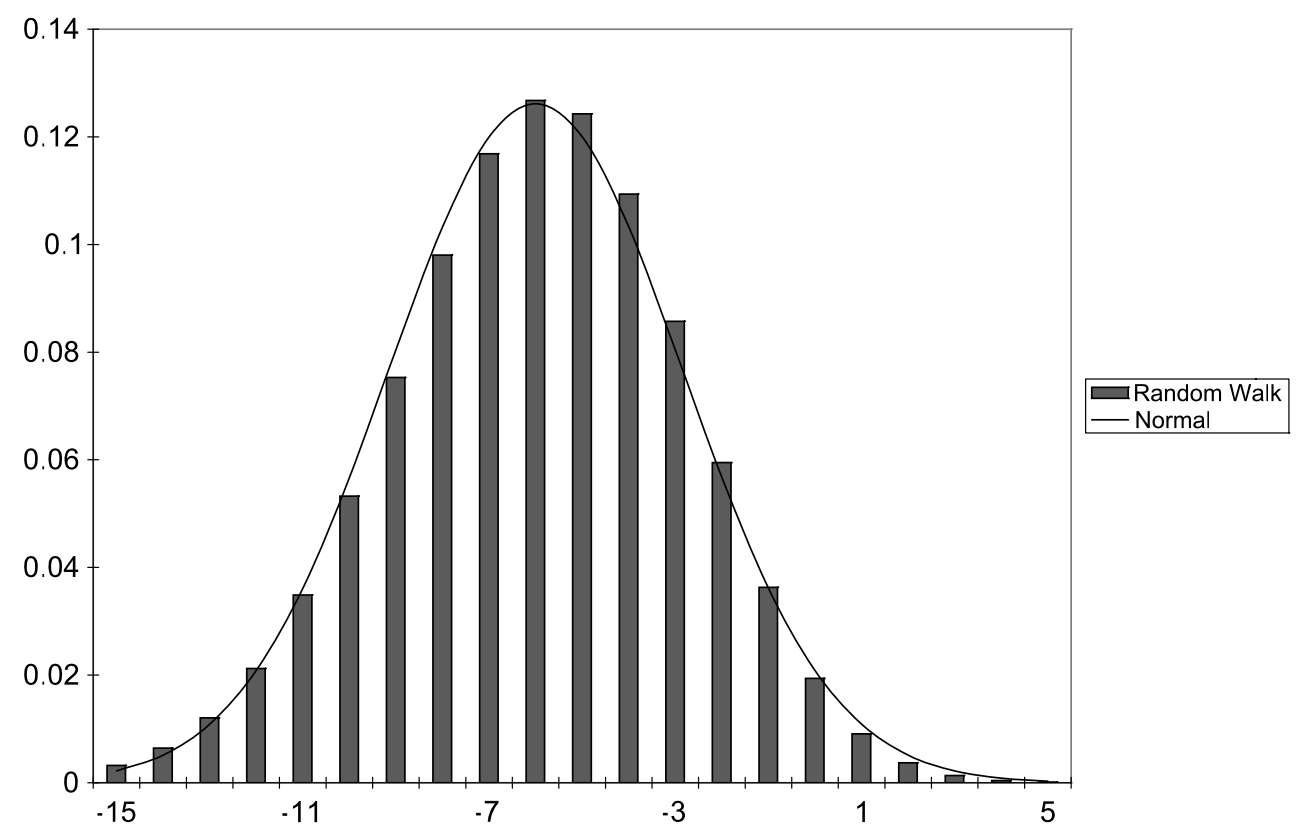

the laws of the generalized random walk, but do not go below zero before time $t$, plus all sample paths that following the laws of the generalized random walk do go below zero prior to time $t$, but for which $Z(t)-\inf _{u \in(s, t)} Z(u)=0$ where $Z(t)$ is the level of the generalized random walk process.

When the $\phi_{k}(s, t)$ are known, we may compute the entire distribution of the quasi-birth-and-death process and also obtain the rising factorial moments. These results are summarized in the following theorem.

Theorem 2 (Time-inhomogeneous QBD transient solution) The transient distribution for the time-inhomogeneous quasi-birth-and-death process with the infinitesimal generator given in (1) is given by

$$
\begin{aligned}
\mathbf{p}_{j}(t)= & \int_{s}^{t}\left[\mathbf { p } _ { 0 } ( v ) \left(\left(\mathbf{B}(v)-\mathbf{A}_{0}(v)\right) \phi_{j}(v, t)\right.\right. \\
& \left.\left.-\mathbf{A}_{-1}(v) \phi_{j+1}(v, t)\right)\right] d v \\
& +\sum_{k=0}^{\infty} \mathbf{p}_{k}(s) \phi_{j-k}(s, t)
\end{aligned}
$$

The rising factorial moments of the $Q B D$ satisfy the equations

$$
\begin{aligned}
& (m-1) ! \mathbf{q}_{0}^{(m)}(t) \\
& =(m-1) ! \int_{s}^{t}\left[\mathbf{p}_{0}(v)\left(\mathbf{B}(v)-\mathbf{A}_{0}(v)\right) \phi_{0}(v, t)\right. \\
& \quad-\mathbf{q}_{0}^{(m)}(v) \mathbf{A}_{-1}(v) \phi_{1}(v, t)
\end{aligned}
$$




$$
\begin{aligned}
& \left.+\sum_{r=1}^{m} \mathbf{q}_{0}^{(r)}(v) \mathbf{A}_{1}(v) \phi_{0}(v, t)\right] d v \\
& +(m-1) ! \sum_{k=0}^{\infty} \mathbf{q}_{k}^{(m)}(s) \phi_{-k}(s, t)
\end{aligned}
$$

and

$$
\begin{aligned}
& m ! \mathbf{q}_{0}^{(m+1)}(t) \\
& =m ! \int_{s}^{t}\left[\mathbf{p}_{0}(v)\left(\mathbf{B}(v)-\mathbf{A}_{0}(v)\right)-\mathbf{q}_{0}^{(m)}(v) \mathbf{A}_{-1}(v)\right. \\
& \left.\quad+\sum_{r=1}^{m} \mathbf{q}_{0}^{(r)}(v) \mathbf{A}_{1}(v)\right] \mathbf{U}(v, t) d v \\
& \quad+m ! \mathbf{q}_{0}^{(m+1)}(s) \mathbf{U}(s, t),
\end{aligned}
$$

where $\mathbf{U}(s, t)$ is an evolution operator such that $\mathbf{U}(t, t)=\mathbf{I}$ and $\frac{\partial}{\partial t} \mathbf{U}(s, t)=\mathbf{U}(s, t) \mathbf{A}(t)$ and $\mathbf{A}(t)=\mathbf{A}_{1}(t)+\mathbf{A}_{0}(t)+$ $\mathbf{A}_{-1}(t)$.

Proof Equation (9) is obtained from the generating function $P_{z}^{(0)}(s, t)$, (5) with $m=0$, by taking the coefficient of $z^{j}$. Equation (10) is obtained from the generating function $P_{z}^{(m)}(s, t)$ by taking the coefficient of $z^{0}$. Equation (11) is obtained from the generating function $P_{z}^{(m)}(s, t)$ by setting $z=1$.

With the solution obtained from (8), we may use (11) to solve for each of the rising factorial moments, recursively.

\subsection{The periodic problem}

If we set $s=t-1$ in (5), we obtain the generating function for the asymptotic periodic solution to the QBD. Recall that $\mathbf{A}_{i}(t), i=-1,0,1$ are each periodic with period one. Then we have

$$
\begin{aligned}
P_{z}^{(m)}(t)= & \int_{t-1}^{t}\left[\mathbf{p}_{0}(v)\left(\mathbf{B}(v)-\mathbf{A}_{0}(v)\right)-z^{-1} \mathbf{q}_{0}^{(m)}(v) \mathbf{A}_{-1}(v)\right. \\
& \left.+\sum_{r=1}^{m} \mathbf{q}_{0}^{(r)}(v) \mathbf{A}_{1}(v)\right] \\
& \times \Phi_{z}(v, t) d v\left[\mathbf{I}-\Phi_{z}(t-1, t)\right]^{-1} .
\end{aligned}
$$

Given $\mathbf{p}_{0}(t)$, and $\phi_{k}(s, t), k=0,1,2, \ldots$ we may find the asymptotic periodic distribution of the QBD and its rising factorial moments. The functions $\phi_{k}(s, t)$ for $k<0$, do not appear in the formulae for the coefficients of $z^{k}, k \geq 0$ and so are not required for finding the asymptotic periodic distribution or its moments.
Theorem 3 (Time-inhomogeneous QBD asymptotic periodic solution) The asymptotic periodic distribution for the time-inhomogeneous quasi-birth-and-death process with the infinitesimal generator given in (1) is given by

$$
\begin{aligned}
\mathbf{p}_{j}(t)= & \int_{t-1}^{t} \mathbf{p}_{0}(v)\left[\left(\mathbf{B}(v)-\mathbf{A}_{0}(v)\right) \sum_{n=0}^{\infty} \phi_{j}(v, t+n)\right. \\
& \left.-\mathbf{A}_{-1}(v) \sum_{n=0}^{\infty} \phi_{j+1}(v, t+n)\right] d v .
\end{aligned}
$$

The rising factorial moments are given by

$$
\begin{aligned}
(m-1) ! \mathbf{q}_{0}^{(m)}(t) & \\
= & (m-1) ! \int_{t-1}^{t}\left[\left(\mathbf{p}_{0}(v)\left(\mathbf{B}(v)-\mathbf{A}_{0}(v)\right)\right.\right. \\
& \left.+\sum_{i=1}^{m} \mathbf{q}_{0}^{(i)}(v) \mathbf{A}_{1}(v)\right) \sum_{n=0}^{\infty} \phi_{0}(v, t+n) \\
& \left.-\mathbf{q}_{0}^{(m)}(v) \mathbf{A}_{-1}(v) \sum_{n=0}^{\infty} \phi_{1}(v, t+n)\right] d v .
\end{aligned}
$$

Proof Equation (13) is obtained from the generating function $P_{z}^{(0)}(s, t),(12)$, by taking the coefficient of $z^{j}$. Equation (14) is obtained from the generating function $P_{z}^{(m)}(s, t)$ by taking the coefficient of $z^{0}$. We have also used the following identities for $\Phi(v, t)$ :

$\left[\mathbf{I}-\Phi_{z}(t-1, t)\right]^{-1}=\sum_{k=0}^{\infty} \Phi_{z}^{k}(t-1, t)=\sum_{k=0}^{\infty} \Phi_{z}(t-k, t)$,

$\Phi_{z}(v, s) \Phi_{z}(s, t)=\Phi_{z}(v, t)$

and

$\Phi_{z}(t-1, t) \Phi_{z}(v, t)=\Phi_{z}(v, t+1)=\Phi_{z}(v-1, t)$.

Let us pause to consider the expression $\sum_{n=0}^{\infty} \phi_{j}(v, t+$ $n)$. Suppose that we are investigating a periodic generalized random walk process with daily period and that $v$ is 3:00 in the afternoon and $t$ is midnight, then $\sum_{n=0}^{\infty} \phi_{j}(\nu, t+n)$ gives the expected number of days (for all time) that the process is in level $j+k$ at midnight given that the process started in level $k$ at 3:00 one afternoon.

An alternative formula for the rising factorial moments is given in the following theorem.

Theorem 4 The rising factorial moments for the periodic $Q B D$ are given by 


$$
\begin{aligned}
& m ! \mathbf{q}_{0}^{(m+1)}(t) \\
& =m ! \int_{t-1}^{t}\left[\left(\mathbf{p}_{0}(v)\left(\mathbf{B}(v)-\mathbf{A}_{0}(v)\right)\right.\right. \\
& \left.\quad+\sum_{i=1}^{m} \mathbf{q}_{0}^{(i)}(v) \mathbf{A}_{1}(v)\right) \sum_{n=0}^{\infty} \sum_{j=0}^{\infty} \phi_{j}(v, t+n) \\
& \left.\quad-\mathbf{q}_{0}^{(m)}(v) \mathbf{A}_{-1}(v) \sum_{n=0}^{\infty} \sum_{j=1}^{\infty} \phi_{j}(v, t+n)\right] d v .
\end{aligned}
$$

Proof Use the generating function, (12), to find $\mathbf{q}_{j}^{(m)}(t)$ and sum these to obtain $\mathbf{q}_{0}^{(m+1)}(t)$.

The asymptotic periodic distribution will exist if the associated generalized random walk has a leftward drift, i.e. if $\sum_{n=0}^{\infty} \sum_{j=0}^{\infty} \phi_{j}(\nu, t+n)<\infty$.

Note that $\mathbf{q}_{0}^{(m+1)}(t)=\lim _{z \rightarrow 1} P_{z}^{(m)}(t)$, so we might naïvely write

$$
\begin{aligned}
\mathbf{q}_{0}^{(m+1)}(t)= & \int_{t-1}^{t}\left[\mathbf{p}_{0}(v)\left(\mathbf{B}(v)-\mathbf{A}_{0}(v)\right)-\mathbf{q}_{0}^{(m)}(v) \mathbf{A}_{-1}(v)\right. \\
& \left.+\sum_{r=1}^{m} \mathbf{q}_{0}^{(r)}(v) \mathbf{A}_{1}(v)\right] \\
& \times \mathbf{U}(v, t) d v[\mathbf{I}-\mathbf{U}(t-1, t)]^{-1}
\end{aligned}
$$

but $\mathbf{I}-\mathbf{U}(t-1, t)$ is singular. When the process is stable and $\mathbf{q}_{0}^{(m+1)}(t)$ is finite, this implies that

$$
\begin{aligned}
& \int_{t-1}^{t}\left[\mathbf{p}_{0}(v)\left(\mathbf{B}(v)-\mathbf{A}_{0}(v)\right)-\mathbf{q}_{0}^{(m)}(v) \mathbf{A}_{-1}(v)\right. \\
& \left.\quad+\sum_{r=1}^{m} \mathbf{q}_{0}^{(r)}(v) \mathbf{A}_{1}(v)\right] \mathbf{U}(v, t) d v \operatorname{adj}(\mathbf{I}-\mathbf{U}(t-1, t))
\end{aligned}
$$

is the zero vector. We may apply l'Hôpital's rule to obtain an alternative expression for the rising factorial moments that requires $\mathbf{p}_{0}(t)$, but does not require a knowledge of the functions $\phi_{j}(v, t+n)$. We summarize this result in the theorem that follows, but first we need some definitions:

$$
\begin{aligned}
& \Psi(s, t)=\left.\frac{d}{d z} \Phi_{z}(s, t)\right|_{z=1} \\
& f(t)=\left.\frac{d}{d z}\left|\mathbf{I}-\Phi_{z}(t-1, t)\right|\right|_{z=1} \\
& \mathbf{G}(t)=\left.\frac{d}{d z} \operatorname{adj}\left(\mathbf{I}-\Phi_{z}(t-1, t)\right)\right|_{z=1} .
\end{aligned}
$$

An integral equation for $\Psi(s, t)$ is

$$
\Psi(s, t)=\int_{s}^{t} \mathbf{U}(s, v)\left(\mathbf{A}_{1}(v)-\mathbf{A}_{-1}(v)\right) \mathbf{U}(v, t) d v .
$$

This equation was obtained by differentiating $\Phi_{z}(s, t)$ as given in (7) by $z$ and setting $z=1$. The functions $f(t)$ and $\mathbf{G}(t)$ may be expressed in terms of the components of the matrices $\mathbf{U}(t-1, t)$ and $\Psi(t-1, t)$. For example, if we have $2 \times 2$ blocks indexed from 0 to 1 , then

$$
\begin{aligned}
f(t)= & -\psi_{00}(t-1, t)-\psi_{11}(t-1, t) \\
& +\psi_{00}(t-1, t) u_{11}(t-1, t) \\
& +\psi_{11}(t-1, t) u_{00}(t-1, t) \\
& -\psi_{01}(t-1, t) u_{10}(t-1, t) \\
& -\psi_{10}(t-1, t) u_{01}(t-1, t)
\end{aligned}
$$

and

$\mathbf{G}(t)=\left[\begin{array}{cc}-\psi_{11}(t-1, t) & \psi_{01}(t-1, t) \\ \psi_{10}(t-1, t) & -\psi_{00}(t-1, t)\end{array}\right]$.

Theorem 5 The rising factorial moments for the periodic $Q B D($ with $f(t) \neq 0)$ are given by

$$
\begin{aligned}
& m ! \mathbf{q}_{0}^{(m+1)}(t) \\
& =\frac{m !}{f(t)}\left[\int_{t-1}^{t} \mathbf{q}_{0}^{(m)}(v) \mathbf{A}_{-1}(v) \mathbf{U}(v, t) d v\right. \\
& \quad \times \operatorname{adj}(\mathbf{I}-\mathbf{U}(t-1, t))+\int_{t-1}^{t}\left(\mathbf{p}_{0}(v)\left(\mathbf{B}(v)-\mathbf{A}_{0}(v)\right)\right. \\
& \left.\quad-\mathbf{q}_{0}^{(m)}(v) \mathbf{A}_{-1}(v)+\sum_{r=1}^{m} \mathbf{q}_{0}^{(r)}(v) \mathbf{A}_{1}(v)\right) \\
& \quad \times(\Psi(v, t) \operatorname{adj}(\mathbf{I}-\mathbf{U}(t-1, t))+\mathbf{U}(v, t) \mathbf{G}(t)) d v] .
\end{aligned}
$$

Proof Apply l'Hôpital's rule to $\lim _{z \rightarrow 1} P_{z}^{(m)}(t)$ and multiply both sides of the equation by $m$ !.

\section{Examples}

\subsection{The $M_{t} / M_{t} / 1$ queue}

For early work on the exact transient solution (up to an integral equation) of the single-server queue with time-varying rates see [15]. Other references include [17, 25] (transient case), and [18] (periodic asymptotic). We derive formulae for the idle probability, the mean and the variance for the general time-varying single server Poisson queue and then apply them to an example used in the recent paper by Zeifman et al. [24].

For this example, the $\mathbf{A}_{i}(t), i=-1,0,1$ and $\mathbf{B}(t)$ are scalars; $\mathbf{A}_{1}(t)=\lambda(t), \mathbf{A}_{0}(t)=-\lambda(t)-\mu(t), \mathbf{A}_{-1}(t)=\mu(t)$ 
and $\mathbf{B}(t)=-\lambda(t)$. Then

$$
\begin{aligned}
\phi_{k}(s, t)= & \sum_{j=0}^{\infty} \frac{\left(\int_{s}^{t} \mu(u) d u\right)^{j}\left(\int_{s}^{t} \lambda(u) d u\right)^{j+k}}{(j+k) ! j !} \\
& \times e^{-\int_{s}^{t} \lambda(u) d u-\int_{s}^{t} \mu(u) d u}
\end{aligned}
$$

for $k \geq 0$ and

$$
\begin{aligned}
\phi_{k}(s, t)= & \sum_{j=0}^{\infty} \frac{\left(\int_{s}^{t} \mu(u) d u\right)^{j-k}\left(\int_{s}^{t} \lambda(u) d u\right)^{j}}{(j-k) ! j !} \\
& \times e^{-\int_{s}^{t} \lambda(u) d u-\int_{s}^{t} \mu(u) d u}
\end{aligned}
$$

for $k<0$. This is the probability of $k$ more steps to the right than to the left during the time interval from $s$ to $t$ for a time-dependent random walk with rates $\lambda(t)$ for steps to the right and $\mu(t)$ for steps to the left. To see this, recall that for a Poisson process, $Z(t)$, with constant parameter $\lambda$, $P\{Z(t)=j\}=e^{-\lambda t} \frac{(\lambda t)^{j}}{j !}$. If the rates are time-varying with parameter $\lambda(t)$, then $P\{Z(t)=j\}=e^{-\int_{0}^{t} \lambda(u) d u} \frac{\left(\int_{0}^{t} \lambda(u) d u\right)^{j}}{j !}$. If we have two such independent processes, the first giving the distribution of the number of steps to the right, and the second with time-varying rate $\mu(t)$ giving the distribution of the number of steps to the left, then the distribution for the net number of steps travelled to the right is given by $\phi_{k}(0, t)$, $k$ integer.

The function $\phi_{k}(s, t)$ may be expressed in terms of modified Bessel functions [6]:

$$
\begin{aligned}
\phi_{k}(s, t)= & \left(\frac{\int_{s}^{t} \lambda(u) d u}{\int_{s}^{t} \mu(u) d u}\right)^{k / 2} \\
& \times I_{k}\left(2 \sqrt{\left(\int_{s}^{t} \lambda(u) d u\right)\left(\int_{s}^{t} \mu(u) d u\right)}\right) \\
& \times e^{-\int_{s}^{t} \lambda(u) d u-\int_{s}^{t} \mu(u) d u} .
\end{aligned}
$$

The rising factorial moments for the transient solution are

$$
\begin{aligned}
m ! q_{0}^{(m+1)}(t)= & m ! \int_{s}^{t}\left[p_{0}(v) \mu(v)-q_{0}^{(m)}(v) \mu(v)\right. \\
& \left.+\sum_{r=1}^{m} q_{0}^{(r)}(v) \lambda(v)\right] d v+m ! q_{0}^{(m+1)}(s) .
\end{aligned}
$$

In particular, for $p_{i}(s)=1$ (when there are known to be $i$ in the system at some initial time $s), q_{0}^{(0)}(t)$ is the solution of the Volterra equation of the second kind:

$$
\begin{aligned}
q_{0}^{(0)}(t)= & p_{0}(t) \\
= & \int_{s}^{t}\left[p_{0}(v) \mu(v)\left(\phi_{0}(v, t)-\phi_{1}(v, t)\right)\right] d v \\
& +\phi_{-i}(s, t) .
\end{aligned}
$$

Furthermore,

$$
\begin{aligned}
& q_{0}^{(1)}(t)=1, \\
& E[X(t)]=\int_{s}^{t} p_{0}(v) \mu(v) d v+\int_{s}^{t}(\lambda(u)-\mu(u)) d u+i,
\end{aligned}
$$

and

$$
\begin{aligned}
\operatorname{Var}[X(t)]= & 2 q_{0}^{(3)}(t)-q_{0}^{(2)}(t)-\left[q_{0}^{(2)}(t)\right]^{2} \\
= & (-2 i-1) \int_{s}^{t} p_{0}(v) \mu(v) d v \\
& +\int_{s}^{t}(\lambda(u)+\mu(u)) d u \\
& +2 \int_{s}^{t}\left[p_{0}(v) \mu(v)\left(\int_{s}^{v}(\mu(u)-\lambda(u)) d u\right)\right] d v \\
& -\left[\int_{s}^{t} p_{0}(v) \mu(v) d v\right]^{2} .
\end{aligned}
$$

Define $\bar{\mu}=\int_{t-1}^{t} \mu(u) d u$ and $\bar{\lambda}=\int_{t-1}^{t} \lambda(u) d u$. The asymptotic periodic moments are given by the formula:

$$
\begin{aligned}
& q_{0}^{(m+1)}(t) \\
& =\frac{1}{\bar{\mu}-\bar{\lambda}} \int_{t-1}^{t}\left[\mu(v) q_{0}^{(m)}(v)+\left(\mu(v)\left(p_{0}(v)-q_{0}^{(m)}(v)\right)\right.\right. \\
& \left.\left.\quad+\lambda(v) \sum_{r=1}^{m} q_{0}^{(r)}(v)\right)\left(\int_{t-1}^{v}(\lambda(u)-\mu(v)) d u\right)\right] d v .
\end{aligned}
$$

We apply (13) to find $p_{0}(t)$ and also use the fact that $q_{0}^{(1)}(t)=1$.

The idle probability satisfies the integral equation

$$
\begin{aligned}
p_{0}(t)= & \int_{t-1}^{t} \mu(v) p_{0}(v) \\
& \times \sum_{n=0}^{\infty}\left(\phi_{0}(v, t+n)-\phi_{1}(v, t+n)\right) d v
\end{aligned}
$$

We can solve this integral equation numerically by discretizing the function $\mu(v) \sum_{n=0}^{\infty}\left(\phi_{0}(v, t+n)-\phi_{1}(v, t+n)\right)$, applying numerical integration weights and finding the eigenvector of the resulting matrix that corresponds to the eigenvalue 1 . This will yield $p_{0}(t)$ up to a constant multiple. We substitute this eigenvector into the equation for $q_{0}^{(1)}(t)$ to scale the eigenvector properly. For $q_{0}^{(1)}(t),(17)$ becomes

$q_{0}^{(1)}(t)=1=\frac{1}{\bar{\mu}-\bar{\lambda}} \int_{t-1}^{t} \mu(v) p_{0}(v) d \nu$.

We illustrate this with an example from Zeifman et al. [24]. Let $\lambda(t)=1+\sin (2 \pi t)$ and $\mu(t)=4+2 \cos (2 \pi t)$. 


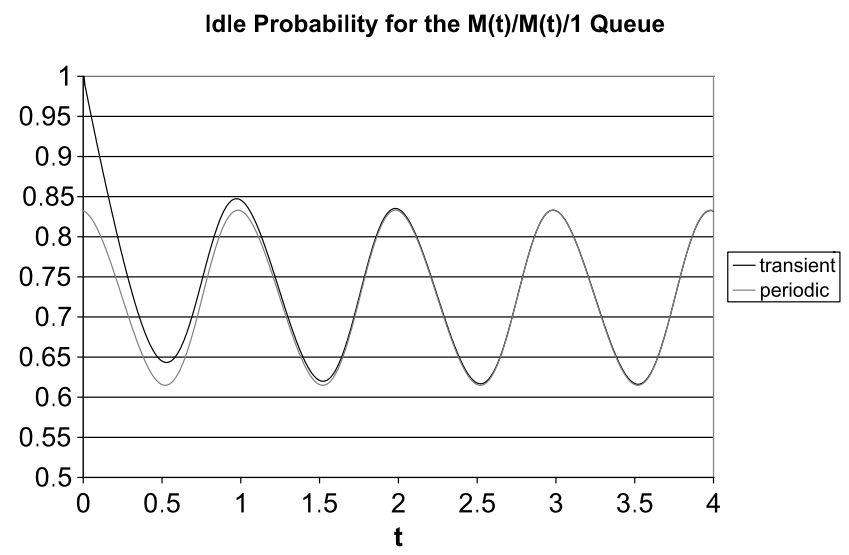

(a) The idle probabilities for the queue

$E[X(t)]$ for the $M(t) / M(t) / 1$ Queue

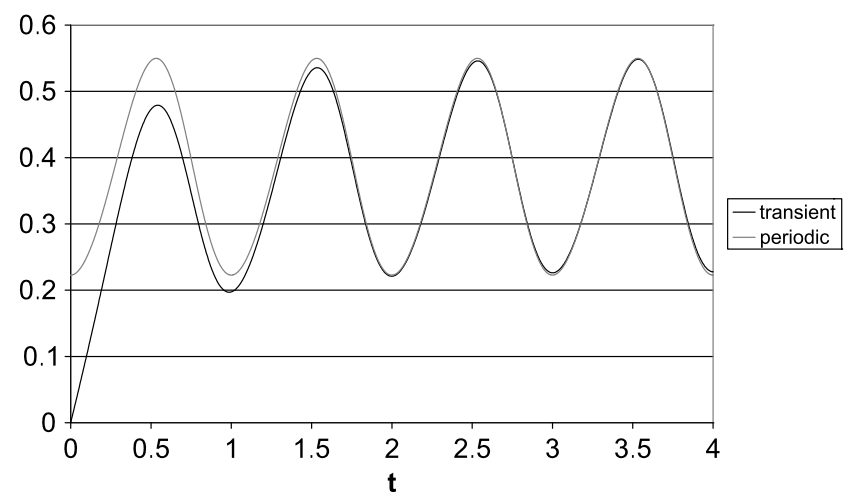

(b) The expected number in the queue

Fig. 2 An $M_{t} / M_{t} / 1$ queue with arrival rate $\lambda(t)=1+\sin (2 \pi t)$, service rate $\mu=4+2 \cos (2 \pi t)$. Transient and asymptotic cases are shown

Figure 2 a shows the asymptotic periodic probability that the queue is idle, and the transient probability given that it was idle at $t=0$. is

The asymptotic periodic expected number in the system

$$
\begin{aligned}
E[X(t)]= & q_{0}^{(2)}(t)-1 \\
= & \frac{\bar{\lambda}}{\bar{\mu}-\bar{\lambda}}+\frac{\bar{\mu}-\bar{\lambda}}{2} \\
& +\frac{\int_{t-1}^{t} \mu(\nu) p_{0}(\nu) \int_{\nu}^{t}(\lambda(\xi)-\mu(\xi)) d \xi d v}{\bar{\mu}-\bar{\lambda}} .
\end{aligned}
$$

This is shown in Fig. 2b. For queues with constant rates, the last two terms cancel one another. The graph shows the asymptotic periodic expected number in the queue, and the transient expected number in queue given that it was idle at $t=0$. The expectation for the constant rate case with $\bar{\lambda}=1$ and $\bar{\mu}=4$ is $1 / 3$. For this time-varying queue, the mean is approximately 0.383 . This is the same result obtained by Zeifman et al. [24] to three decimal places of accuracy. These computations were done using 3rd order Newton-
Gregory integration weights with a mesh size of 0.00125 for the periodic solution. A mesh of 0.0025 was used for the transient problem. The Newton-Gregory quadrature rules are a convenient numerical method for solving Volterra equations of the second kind [21] such as the equations that arise in solving the transient problem. Any quadrature method with equally spaced nodes will work for obtaining the asymptotic periodic distribution and its moments.

\subsection{The $M_{t} / M_{t} / c$ queue}

For the $M_{t} / M_{t} / c$ queue,

$\phi_{k}(t)$

$$
=\left[\begin{array}{cccc}
\tilde{I}_{k c}(s, t) & \tilde{I}_{k c+1}(s, t) & \cdots & \tilde{I}_{k c+c-1}(s, t) \\
\tilde{I}_{k c-1}(s, t) & \tilde{I}_{k c}(s, t) & \cdots & \tilde{I}_{k c+c-2}(s, t) \\
\vdots & \ddots & \ddots & \vdots \\
\tilde{I}_{k c-c+1}(s, t) & \tilde{I}_{k c-c+2}(s, t) & \cdots & \tilde{I}_{k c}(s, t)
\end{array}\right],
$$

where

$$
\begin{aligned}
\tilde{I}_{k}(s, t)= & \sum_{j=0}^{\infty} \frac{\left(c \int_{s}^{t} \mu_{i}(u) d u\right)^{j}\left(\int_{s}^{t} \lambda_{i}(u) d u\right)^{j+k}}{(j+k) ! j !} \\
& \times e^{-\int_{s}^{t} \lambda_{i}(u) d u-c \int_{s}^{t} \mu_{i}(u) d u}
\end{aligned}
$$

for $k \geq 0$ and

$$
\begin{aligned}
\tilde{I}_{k}(s, t)= & \sum_{j=0}^{\infty} \frac{\left(c \int_{s}^{t} \mu_{i}(u) d u\right)^{j-k}\left(\int_{s}^{t} \lambda_{i}(u) d u\right)^{j}}{(j-k) ! j !} \\
& \times e^{-\int_{s}^{t} \lambda_{i}(u) d u-c \int_{s}^{t} \mu_{i}(u) d u}
\end{aligned}
$$

for $k<0$ give the transition probabilities for a random walk with time-varying transition rates $\lambda(t)$ for a step to the left and $c \mu(t)$ for a step to the right. The $i$ th component of the vector $\mathbf{p}_{0}(t)$ will give the probability of $i-1$ in the system for $i=1, \ldots, c$, and more generally, the $i$ th component of the vector $\mathbf{p}_{k}(t)$ will give the probability that there are $c k+i-1$ in the system (waiting or in service). The $i$ th component of the vector $\mathbf{q}_{0}^{(1)}(t)$ gives the probability that the number in the system is equal to $c k+i$ for some nonnegative integer $k$. Solving for performance measures for the time-varying multi-server queue can be done more efficiently using methods outlined in [17] and [18] which involve scalar integral functions, but we provide a numerical example for the $M_{t} / M_{t} / 2$ queue using the method of this paper for the purpose of illustration.

Consider the $M_{t} / M_{t} / 2$ queue with $\lambda(t)=1+\sin (2 \pi t)$ and $\mu(t)=4+2 \cos (2 \pi t)$. The average expected number in the queue is found to be 0.2946 using numerical integration as described in the previous example. This equals the result given in the Zeifman paper [24] to four decimal places of accuracy. The probability that there are no customers in service 
Idle Probability for the $M(t) / M(t) / 2$ Queue

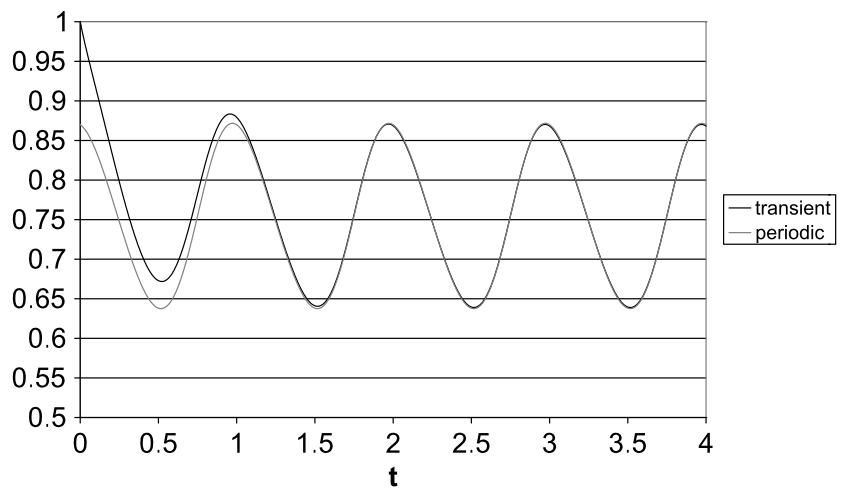

(a) The idle probabilities for the queue

$E[X(t)]$ for the $M(t) / M(t) / 2$ Queue

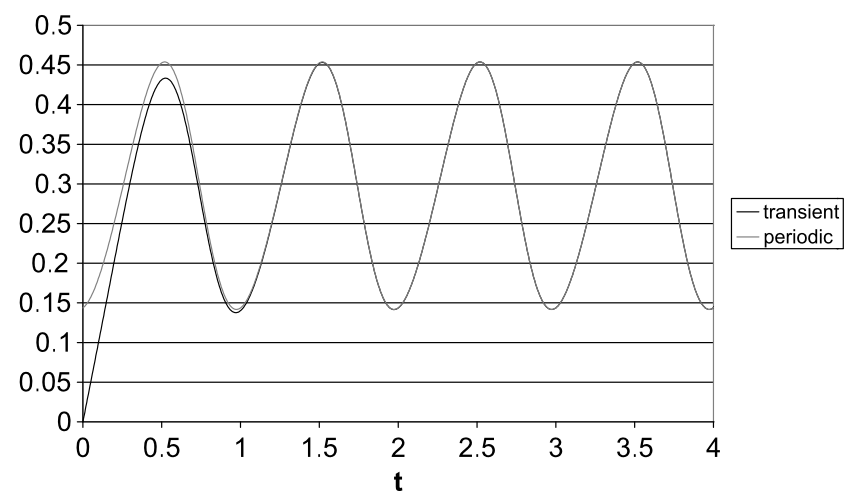

(b) The expected number in the queue

Fig. 3 An $M_{t} / M_{t} / 2$ queue with arrival rate $\lambda(t)=1+\sin (2 \pi t)$, service rate $\mu=4+2 \cos (2 \pi t)$. Transient and asymptotic cases are shown

is graphed in Fig. 3a and the expected number in the queue for both transient and asymptotic periodic cases is graphed in Fig. 3b.

\section{3 $P H_{t} / M_{t} / 1$ queue with $j$-Erlang interarrival times}

We consider a time-varying parameter version of an $E_{j} / M / 1$ queue, (see pp. 12-15, [14]). For this example,

$$
\begin{aligned}
\mathbf{B}(t) & =\left[\begin{array}{cccc}
-v(t) & \cdot & \cdot & \cdot \\
v(t) & -v(t) & \cdot & \cdot \\
\vdots & \ddots & \ddots & \vdots \\
\cdot & & v(t) & -v(t)
\end{array}\right], \\
\mathbf{A}_{1}(t) & =\left[\begin{array}{cccc}
\cdot & \cdots & \cdot & v(t) \\
\cdot & \cdots & \ldots & \cdot \\
\vdots & \vdots & \vdots & \vdots \\
\cdot & \cdots & \ldots & \cdot
\end{array}\right]
\end{aligned}
$$

$\mathbf{A}_{0}(t)$

$$
\begin{aligned}
& =\left[\begin{array}{cccc}
-v(t)-\mu(t) & \cdot & \cdot & \cdot \\
v(t) & -v(t)-\mu(t) & \cdot & \cdot \\
\cdot & \ddots & \ddots & \cdot \\
\cdot & \cdot & v(t) & -v(t)-\mu(t)
\end{array}\right], \\
& \mathbf{A}_{-1}(t)=\left[\begin{array}{ccc}
\mu(t) & \cdot & \cdot \\
\cdot & \ddots & \cdot \\
\cdot & \cdot & \mu(t)
\end{array}\right],
\end{aligned}
$$

and

$$
\begin{aligned}
& \Phi_{z}(s, t) \\
& =e^{\int_{s}^{t}\left(\left(z^{-1}-1\right) \mu(u)-v(u)\right) d u} \\
& \quad \times\left[\begin{array}{cccc}
f_{z}^{(0)}(s, t) & f_{z}^{(-1)}(s, t) & \ldots & f_{z}^{(-j+1)}(s, t) \\
f_{z}^{(1)}(s, t) & f_{z}^{(0)}(s, t) & \ldots & f_{z}^{(-j+2)}(s, t) \\
\vdots & \ddots & \ddots & \vdots \\
f_{z}^{(j-1)}(s, t) & f_{z}^{(j-2)}(s, t) & \ldots & f_{z}^{(0)}(s, t)
\end{array}\right],
\end{aligned}
$$

where

$f_{z}^{(n)}(s, t)=\sum_{m=n}^{\infty} \frac{z^{m}\left(\int_{s}^{t} v(u) d u\right)^{j m-n}}{(j m-n) !}$

for $n<0$ and

$f_{z}^{(n)}(s, t)=\sum_{m=0}^{\infty} \frac{z^{m}\left(\int_{s}^{t} v(u) d u\right)^{j m-n}}{(j m-n) !}$

for $n \geq 0$. The related random walk transition probabilities are then given by the Toeplitz matrix

$\phi_{k}(s, t)$

$$
\begin{aligned}
= & e^{-\int_{s}^{t}(\mu(u)+v(u)) d u} \\
& \times\left[\begin{array}{cccc}
g^{(0)}(k, s, t) & g^{(-1)}(k, s, t) & \ldots & g^{(-j+1)}(k, s, t) \\
g^{(1)}(k, s, t) & g^{(0)}(k, s, t) & \ldots & g^{(-j+2)}(k, s, t) \\
\vdots & \ddots & \ddots & \vdots \\
g^{(j-1)}(k, s, t) & g^{(j-2)}(k, s, t) & \ldots & g^{(0)}(k, s, t)
\end{array}\right],
\end{aligned}
$$

where

$g^{(n)}(k, s, t)=\sum_{m=0 \wedge-k}^{\infty} \frac{\left(\int_{s}^{t} v(u) d u\right)^{j(m+k)+n}\left(\int_{s}^{t} \mu(u) d u\right)^{m}}{(j(m+k)+n) ! m !}$

for $n \geq 0$ and

$g^{(n)}(k, s, t)=\sum_{m=n-j}^{\infty} \frac{\left(\int_{s}^{t} v(u) d u\right)^{j(m+k)-n}\left(\int_{s}^{t} \mu(u) d u\right)^{m}}{(j(m+k)-n) ! m !}$

for $n<0$. 


\section{Estimating $\phi_{k}(s, t)$ using the product integral approach}

The description of the product integral presented here is based upon the overview by Richard Gill for biostatisticians [4]. The product integral was developed by Vito Volterra as a tool in the solution of a differential equation of the form

$\frac{\partial}{\partial t} \mathbf{F}(s, t)=\mathbf{F}(s, t) \mathbf{A}(t)$

with $F(s, s)=\mathbf{I}$ or equivalently, the integral equation

$\mathbf{F}(s, t)=\mathbf{I}+\int_{s}^{t} \mathbf{F}(s, u) \mathbf{A}(u) d u$

where the unknown $\mathbf{F}(s, t)$, and the known $\mathbf{A}(t)$ are matrix valued functions. Ordinary integration may be thought of as summation of very many mostly very small terms. In a similar way, product integration may be thought of as the product of very many terms. For scalar product integration the terms will be very close to one. For matrix product integration, the terms will be very close to the identity matrix.

The generating function for the generalized random walk satisfies an integral equation of this form:

$\Phi_{z}(s, t)=\mathbf{I}+\int_{s}^{t} \Phi_{z}(s, u) \mathbf{A}_{z}(u) d u$

where $\mathbf{A}_{z}(t)=z^{-1} \mathbf{A}_{-1}(t)+\mathbf{A}_{0}(t)+z \mathbf{A}_{1}(t)$. In Sect. 2, we expressed $\Phi_{z}(s, t)$ as a Péano series and in terms of the coefficients on $z^{k}$ for $k$ integer. We can also express $\Phi_{z}(s, t)$ as a product integral and this representation can be used to compute $\Phi_{z}(s, t)$ numerically.

Define $\mathbf{Y}(t)=\int_{s}^{t} \mathbf{A}_{z}(u) d u$. Each component of $\mathbf{Y}$ is right continuous with left hand limits. The product integral of $\mathbf{Y}$ over $[s, t]$ is defined as

$$
\begin{aligned}
\prod_{s}^{t}(\mathbf{I}+d \mathbf{Y}(u)) & =\lim _{\max \left|t_{i}-t_{i-1}\right| \rightarrow 0} \prod_{i=1}^{n}\left(\mathbf{I}+\mathbf{Y}\left(t_{i}\right)-\mathbf{Y}\left(t_{i-1}\right)\right) \\
& =\lim _{\max \left|t_{i}-t_{i-1}\right| \rightarrow 0} \prod_{i=1}^{n}\left(\mathbf{I}+\int_{t_{i-1}}^{t} \mathbf{A}_{z}(u) d u\right),
\end{aligned}
$$

where the limit is taken over ever finer partitions $s=t_{0}<$ $t_{1}<\cdots<t_{n}=t$ of the time interval $[s, t]$. For the limit to exist, $\mathbf{Y}$ must be of bounded variation. The generalized random walk generating function may be expressed as the product integral

$\Phi_{z}(s, t)=\prod_{s}^{t}(\mathbf{I}+d \mathbf{Y}(u))$.

If we approximate the solution of a system of differential equations of the form given in (19), then this product integral approach is just an application of the first-order Euler method for solving a system of differential equations. For the random walk generating function, we must obtain the solution of the differential equation in the form of estimates of the coefficient matrices for each power of $z$.

The generator for the generalized random walk is given by

$\mathbf{Q}(t)=\left[\begin{array}{ccccccc}\ddots & \ddots & \ddots & \ddots & \cdot & \cdot & \cdot \\ \cdot & \mathbf{A}_{-1}(t) & \mathbf{A}_{0}(t) & \mathbf{A}_{1}(t) & \cdot & \cdot & \cdot \\ \cdot & \cdot & \mathbf{A}_{-1}(t) & \mathbf{A}_{0}(t) & \mathbf{A}_{1}(t) & \cdot & \cdot \\ \cdot & \cdot & \cdot & \mathbf{A}_{-1}(t) & \mathbf{A}_{0}(t) & \mathbf{A}_{1}(t) & \cdot \\ \cdot & \cdot & \cdot & \cdot & \ddots & \ddots & \ddots\end{array}\right]$,

where its block rows and columns are indexed from minus infinity to infinity and row $i$ is identical to row $i+1$ except that it is shifted one block to the right.

The product integral formula, cannot be applied directly since the matrix $\mathbf{Q}(t)$ is infinite; however, if we apply the product integral formula to the finite matrix $\mathbf{A}_{z}(t)$, the coefficient on $z^{k}$ will be the component in block $(j, j+k)$ of the transition probability matrix for the generalized random walk, (i.e. it will be equal to $\phi_{k}$ ).

We work with the matrices

$\mathbf{A}_{i}(t)=\left[\begin{array}{cccc}\alpha_{0,0}^{(i)}(t) & \alpha_{0,1}^{(i)}(t) & \cdots & \alpha_{0, K-1}^{(i)}(t) \\ \alpha_{1,0}^{(i)}(t) & \alpha_{1,1}^{(i)}(t) & \cdots & \alpha_{1, K-1}^{(i)}(t) \\ \vdots & \vdots & \vdots & \vdots \\ \alpha_{K-1,0}^{(i)}(t) & \alpha_{K-1,1}^{(i)}(t) & \cdots & \alpha_{K-1, K-1}^{(i)}(t)\end{array}\right]$

for $i=-1,0,1$. The probability that a transition occurs in the time interval $(t, t+h)$ from level $j$ to level $j+i$ and from phase $k$ to phase $m$ is approximately $\int_{t}^{t+h} \alpha_{k, m}^{(i)}(u) d u$. The probability that no transition occurs is approximately $1-\sum_{l=0}^{K-1} \int_{t}^{t+h} \alpha_{l}^{(-1)}(u) d u-\sum_{l=0}^{K-1} \int_{t}^{t+h} \alpha_{l}^{(1)}(u) d u-$ $\sum_{l \neq k} \int_{t}^{t+h} \alpha_{l}^{(0)}(u) d u$.

We store our approximation for $\Phi_{z}(s, t)$ in a vector of coefficient blocks. If the matrices $\mathbf{A}_{i}(t)$ are continuous and the mesh is fairly fine $\Phi_{z}\left(t_{j-1}, t_{j}\right) \approx z^{-1} h \mathbf{A}_{-1}\left(t_{j}\right)+\mathbf{I}+$ $h \mathbf{A}_{0}\left(t_{j}\right)+z h \mathbf{A}_{1}\left(t_{j}\right)$ where $h=t_{j}-t_{j-1}$. If the $\mathbf{A}_{i}(t)$ matrices have jumps and the mesh is fairly coarse, we may prefer to use the approximation $\Phi_{z}\left(t_{j-1}, t_{j}\right) \approx z^{-1} \int_{t_{j-1}}^{t_{j}} \mathbf{A}_{-1}(u) d u$ $+\mathbf{I}+\int_{t_{j-1}}^{t_{j}} \mathbf{A}_{0}(u) d u+z \int_{t_{j-1}}^{t_{j}} \mathbf{A}_{1}(u) d u=\mathbf{I}+\int_{t_{j-1}}^{t_{j}} \mathbf{A}_{z}(u) d u$.

Given $\Phi_{z}\left(t_{m}, t_{n}\right) \approx \sum_{k=-(n-m)}^{n-m} z^{k} \mathbf{C}_{k}\left(t_{m}, t_{n}\right)$, we may compute the approximation

$$
\begin{aligned}
\Phi_{z}\left(t_{m}, t_{n+1}\right) \approx & \sum_{k=-(n-m+1)}^{n-m+1} z^{k}\left(\mathbf{C}_{k+1}\left(t_{m}, t_{n}\right) \mathbf{C}_{-1}\left(t_{n}, t_{n+1}\right) .\right. \\
& +\mathbf{C}_{k}\left(t_{m}, t_{n}\right) \mathbf{C}_{0}\left(t_{n}, t_{n+1}\right) \\
& \left.+\mathbf{C}_{k-1}\left(t_{m}, t_{n}\right) \mathbf{C}_{1}\left(t_{n}, t_{n+1}\right)\right) .
\end{aligned}
$$

We approximate the random walk transition probability $\phi_{k}\left(t_{m}, t_{n}\right)$ with the matrix $\mathbf{C}_{k}\left(t_{m}, t_{n}\right)$. 


\section{Conclusion}

This method provides general formulae for the quasi-birthdeath process with time-varying rates. When the rates are periodic, numerical estimates may be calculated using one period of data. Specific examples are given for which explicit formulae for integral kernels are available. A product integral approach can be used to estimate these kernels when explicit formulae are not known. Future work will extend the $R$ and $G$ functions of matrix analytic methods to a timeinhomogeneous setting $[14,19]$.

Acknowledgements The author thanks two anonymous referees for their suggestions which have improved the presentation of this paper.

\section{References}

1. Breuer, L.: The periodic $B M A P / P H / c$ queue. Queueing Syst. 38, 67-76 (2001)

2. Fadiloglu, M.M., Yeralan, S.: Models of production lines as quasibirth-death processes. Math. Comput. Model. 35, 913-930 (2002)

3. Gikhman, I., Skorokhod, A.: Introduction to the Theory of Random Processes. Saunders, Philadelphia (1969)

4. Gill, R.D.: Product integration. In: Encyclopedia of Biostatistics V, pp. 3530-3534. Wiley, New York (1997)

5. Gill, R.D., Johansen, S.: A survey of product-integration with a view toward application in survival analysis. Ann. Stat. 18, 15011555 (1990)

6. Gradshteyn, I.S., Ryzhik, I.M.: In: Jeffries, A. (ed.) Table of Integrals, Series, and Products, 5th edn. Academic Press, New York (1994)

7. Graham, R.L., Knuth, D.E., Patashnik, O.: Concrete Mathematics, 2nd edn. Addison-Wesley, Reading (1994)

8. Granovsky, B.L., Zeifman, A.: Nonstationary queues: estimation of the rate of convergence. Queueing Syst. 46, 363-388 (2004)

9. Irvani, S.M.R., Luangkesorn, K.L., Simchi-Levi, D.: On assemble to order systems with flexible customers. IIE Trans. 35, 389-403 (2003)

10. Kim, Y.Y., Li, S.: Performance evaluation of packet data services over cellular voice networks. Wirel. Netw. 5, 211-219 (1999)
11. Knuth, D.E.: Two notes on notation. Am. Math. Mon. 99, 403-422 (1992)

12. Kolmogorov, A.N.: Sur le probleme d'attente (French). Mat. Sbornik 38, 101 (1931)

13. Kolmogorov, A.N.: The waiting problem, In: Shiryayev, A.N. (ed.) Selected Works of A.N. Kolmogorov. Probability Theory and Mathematical Statistics, vol. II, pp. 109-114. Kluwer Academic, Boston (1991), translated by G. Lindquist

14. Latouche, G., Ramaswami, V.: Introduction to Matrix Analytic Methods in Stochastic Modeling. American Statistical Association and the Society for Industrial and Applied Mathematics, Philadelphia (1999)

15. Leese, E.L., Boyd, D.W.: Numerical methods of determining the transient behavior of queues with variable arrival rates. J. Can. Oper. Res. Soc. 4, 1-13 (1966)

16. Mandelbaum, A., Massey, W.A.: Strong approximations for timedependent queues. Math. Oper. Res. 20, 33-64 (1995)

17. Margolius, B.H.: Transient solution to the time-dependent multiserver Poisson queue. J. Appl. Probab. 42, 766 (2005)

18. Margolius, B.H.: Periodic solution to the time-inhomogeneous multi-server Poisson queue. Oper. Res. Lett. 35, 125-138 (2007)

19. Neuts, M.F.: Matrix-Geometric Solutions in Stochastic ModelsAn Algorithmic Approach. Dover, New York (1981)

20. Sloane, N.J.A.: The On-Line Encyclopedia of Integer Sequences, (2005) published electronically at http://www research.att.com/ $\sim$ njas/sequences/. The specific sequence is: http://www research. att.com/projects/OEIS?Anum=A008275

21. Wolkenfelt, P.H.M.: On the relation between the repetition factor and numerical stability of direct quadrature methods for second kind Volterra integral equations. SIAM J. Numer. Anal. 20, 10491061 (1983)

22. Yin, G., Zhang, H.: Two-time-scale Markov chains and applications to quasi-birth-death queues. SIAM J. Appl. Math. 65, 567$586(2005)$

23. Yoon, S., Lewis, M.E.: Optimal pricing and admission control in a queueing system with periodically varying parameters. Queueing Syst. 47, 177-199 (2004)

24. Zeifman, A., Leorato, S., Orsingher, E., Satin, Ya., Shilova, G.: Some universal limits for nonhomogeneous birth and death processes. Queueing Syst. 52, 139-151 (2006)

25. Zhang, J., Coyle, E.J.: The transient solution of time-dependent M/M/1 queues. IEEE Trans. Inf. Theory 37, 1690-1696 (1991) 